\title{
Immunotherapy for Non-melanoma Skin Cancer
}

\author{
Sophia Z. Shalhout ${ }^{1}$ [ $\cdot$ Kevin S. Emerick ${ }^{4} \cdot$ Howard L. Kaufman $^{3} \cdot$ David M. Miller $^{1,2}$
}

Accepted: 31 May 2021 / Published online: 27 August 2021

(c) The Author(s), under exclusive licence to Springer Science+Business Media, LLC, part of Springer Nature 2021

\begin{abstract}
Purpose of Review The therapeutic landscape for non-melanoma skin cancer (NMSC) has recently expanded with the development of effective and targeted immunotherapy. Here, we provide an overview of the role of immunotherapy in the management of advanced cutaneous carcinomas.

Recent Findings Several agents were recently U.S. Food and Drug Administration (FDA)-approved for the treatment of locally advanced and metastatic cutaneous squamous cell carcinoma, Merkel cell carcinoma, and basal cell carcinoma. However, recent approvals in tissue-agnostic indications may also benefit other NMSCs including cutaneous adnexal solid tumors with high tumor mutation burdens or microsatellite instability. Furthermore, while FDA-approved indications will likely continue to expand, continued studies are needed to support the role of immunotherapy in the neoadjuvant, adjuvant, and refractory settings.

Summary Immunotherapy is emerging as the standard of care for several advanced NMSCs not amenable to surgery and radiation. Ongoing evaluation of the clinical trial landscape is needed to optimize enrollment and ensure continued innovation.
\end{abstract}

Keywords Immunotherapy $\cdot$ Basal cell carcinoma $\cdot$ Merkel cell carcinoma $\cdot$ Cutaneous squamous cell carcinoma $\cdot$ NMSC . FDA · Cutaneous adnexal carcinomas · Immune checkpoint blockade · Anti-PD-L1 · Anti-CTLA-4 · Anti-PD-1 · Nonmelanoma skin cancer · Clinical trials · Immune checkpoint inhibitors · MCC · BCC · CSCC · Avelumab · Cemiplimab · Pembrolizumab $\cdot$ Nivolumab $\cdot$ Ipilimumab $\cdot$ Adjuvant trials $\cdot$ Neoadjuvant trials $\cdot$ Real-world evidence $\cdot$ Regulatory approvals $\cdot$ Accelerated approvals $\cdot$ Hedgehog inhibitors

This article is part of the Topical Collection on Melanoma

David M. Miller

dmiller4@mgh.harvard.edu

Sophia Z. Shalhout

sshalhout@mgh.harvard.edu

Kevin S. Emerick

kevin_emerick@meei.harvard.edu

Howard L. Kaufman

hlkaufman@mgh.harvard.edu

1 Department of Medicine, Division of Hematology/Oncology, Massachusetts General Hospital, Harvard Medical School, Bartlett Hall, Room 132, 15 Parkman St, Boston, MA 02114, USA

2 Department of Dermatology, Massachusetts General Hospital, Harvard Medical School, Bartlett Hall, Room 132, 15 Parkman St, Boston, MA 02114, USA

3 Department of Surgery, Massachusetts General Hospital, Harvard Medical School, Boston, MA, USA

4 Department of Otolaryngology, Massachusetts Eye and Ear Infirmary, Harvard Medical School, Boston, MA, USA

$\begin{array}{ll}\text { Abbreviations } \\ \text { AE } & \text { Adverse events } \\ \text { BLA } & \text { Biologic license application } \\ \text { BCC } & \text { Basal cell carcinoma } \\ \text { CACs } & \text { Cutaneous adnexal carcinomas } \\ \text { CSCC } & \text { Cutaneous squamous cell carcinoma } \\ \text { CTLA-4 } & \text { Cytotoxic T-lymphocyte-associated antigen 4 } \\ \text { ECOG PS } & \text { Eastern Cooperative Oncology Group Perfor- } \\ & \text { mance Status } \\ \text { FDA } & \text { USA Food and Drug Administration } \\ \text { H\&N } & \text { Head and neck } \\ \text { HHI } & \text { Hedgehog inhibitor } \\ \text { HIV } & \text { Human immunodeficiency virus } \\ \text { ICI } & \text { Immune checkpoint inhibitor } \\ \text { irAE } & \text { Immune-related adverse event } \\ \text { MCC } & \text { Merkel cell carcinoma } \\ \text { MCPyV } & \text { Merkel cell polyomavirus } \\ \text { MMR } & \text { Mismatch repair } \\ \text { MSI } & \text { Microsatellite instability } \\ \text { NDA } & \text { New drug application } \\ \text { NMSC } & \text { Non-melanoma skin cancer }\end{array}$




$\begin{array}{ll}\text { pCR } & \text { Pathological complete response } \\ \text { PFS } & \text { Progression-free survival } \\ \text { PD-L1 } & \text { Programmed death-ligand 1 } \\ \text { PD-1 } & \text { Programmed cell death 1 protein } \\ \text { RWE } & \text { Real world evidence } \\ \text { SOTRs } & \text { Solid organ transplant recipients } \\ \text { TMB } & \text { Tumor mutation burden }\end{array}$

\section{Introduction}

Non-melanoma skin cancers (NMSCs) are exceedingly common, accounting for $30 \%$ of all cancer diagnoses. With an aging population that reflects high levels of cumulative ultraviolet exposure and immunosenescence, the incidence of NMSCs continues to rise [1]. NMSC comprises a heterogeneous group of malignancies including basal cell carcinoma (BCC), cutaneous squamous cell carcinoma (CSCC), Merkel cell carcinoma (MCC), and cutaneous adnexal tumors. Overall mortality is proportionally low, despite high incidence; however, the absolute number of deaths is comparable to melanoma [1]. BCC and CSCC rarely present as locally advanced or metastatic, as the majority present as localized tumors and are treated with curative surgery or radiotherapy. Furthermore, while MCC is one of the most aggressive NMSCs, with nodal and/or distant metastasis detected at presentation in one third of patients, it is a rare cancer [2].

In unresectable locally advanced or metastatic NMSC, systemic therapy may be indicated. Within the last decade, clinical benefit with immune checkpoint inhibitors (ICI) have supported U.S. Food and Drug Administration (FDA) approvals for advanced MCC, CSCC, and most recently, $\mathrm{BCC}$. The aim of this review is to provide an update on the regulatory approvals for immunotherapy in the treatment of advanced NMSC with a focus on cutaneous carcinomas, including MCC, BCC, and CSCC as well as uncommon skin adnexal tumors such as sebaceous carcinoma and porocarcinoma. We also examine ongoing trials and real-world evidence investigating the role of ICIs in the adjuvant, neoadjuvant, and refractory settings of advanced NMSCs and discuss challenges and prospects in the field. Other NMSCs such as cutaneous lymphoma, angiosarcoma, and Kaposi's sarcoma are beyond the scope of this review.

\section{FDA-approved Front-line Immunotherapy in NMSCs}

\section{Cutaneous Squamous Cell Carcinoma}

CSCC is the second most common skin cancer following $\mathrm{BCC}$ and maintains tumor features predictive of response to ICI therapy, including high tumor mutational burden
(TMB) and over representation among immunosuppressed patients [3]. With high mortality rates in patients with metastatic disease, the therapeutic potential of ICI in advanced CSCC gained early considerable interest. Initial reports of clinical benefit and response were described in limited case series and case reports $[4,5]$. Following results of the EMPOWER CSCC-1 phase II (NCT02760498) and phase I (NCT02383212) pivotal trials, the FDA granted regular approval for the anti-PD-1 agent, cemiplimab, in September of 2018 for the treatment of locally advanced or metastatic CSCC in patients deemed ineligible for curative surgery or curative radiation (Table 1) https://www.acces sdata.fda.gov/scripts/cder/daf/index.cfm?event=overview. process\&ApplNo $=761097$. Solid organ transplant recipients (SOTRs), patients who previously received ICI, those requiring immunosuppressants for autoimmune conditions, hepatitis/HIV infected, and patients with an ECOG $\mathrm{PS} \geq 2$ were excluded from enrollment.https://www.acces sdata.fda.gov/scripts/cder/daf/index.cfm?event=overview. process $\& A p p l N o=761097,6 \bullet \bullet]$ An objective response rate (ORR) of $~ 50 \%$ was achieved with $7 \%$ of the phase II cohort achieving a complete response (CR). Cemiplimab was well tolerated with treatment cessation reported in only $7 \%$ and an adverse events (AEs) profile similar to other anti-PD-1 agents.[6・•] Recent updates from the EMPOWER-CSCC-1 and NCT02383212 trials reveal continued durable response and favorable safety profiles [7, 8].

In June of 2020, the FDA approved pembrolizumab, an anti-PD-1 agent, for the treatment of patients with recurrent or metastatic CSCC not amenable to curative surgery or radiation, based on the results of the KEYNOTE-629 (NCT03284424) pivotal trial (Table 1) https://www. accessdata.fda.gov/scripts/cder/daf/index.cfm?event= overview.process\& ApplNo $=125514,9 \bullet]$. An ORR of $34.3 \%$ (95\% CI, 25.3-44.2\%) was reported with $4 \%$ of the cohort achieving CR and a disease control rate of $52.4 \%$ (95\% CI, 42.4-62.2\%).[9•] Eighty-seven percent of the KEYNOTE-629 cohort received one or more previous lines of therapy. In keeping with clinical benefit observed in KEYNOTE-629, initial results from the CARSKIN (NCT02883556) trial where pembrolizumab is being evaluated as first-line monotherapy (e.g., chemotherapy-naïve) demonstrate an ORR of $41 \%$ (95\% CI, 26-58\%) (Table 2) [10].

Retrospective studies of real-world assessment of response to immunotherapy, including pembrolizumab, cemiplimab, and nivolumab, offer insight into the patient population deemed trial ineligible, including solid organ transplant recipients (SOTRs) and patients with autoimmune conditions. ORR ( $31.5 \%$ to $58.7 \%$ ) in the real-world setting appear comparable to trial results, independent of type and line of immunotherapy, and patient immunosuppression status [11-13]. However, a higher ECOG PS at baseline was 
Table 1 FDA-approved agents for NMSCs and tissue-agnostic approvals

\begin{tabular}{|c|c|c|c|c|c|}
\hline Skin cancer, indication & Therapeutic & Mechanism & $\begin{array}{l}\text { Subjects } \\
\text { enrolled }\end{array}$ & Approval date & BLA/NDA \\
\hline \multicolumn{6}{|l|}{ BCC } \\
\hline Superficial BCC & Fluorouracil & Anti-metabolite & 54 & $1975-06-30$ & NDA016831 \\
\hline Superficial BCC & Imiquimod & TLR agonist & 364 & 2004-07-14 & NDA020723 \\
\hline Locally advanced/metastatic BCC & Vismodegib & Hedgehog inhibitor & 96 & 2012-01-30 & NDA203388 \\
\hline Locally advanced BCC & Sonidegib phosphate & Smoothened inhibitor & 194 & $2015-07-24$ & NDA205266 \\
\hline Locally advanced/metastatic BCC, refractory setting & Cemiplimab-RWLC & PD-1 targeted antibody & 112 & 2021-02-09 & BLA761097 \\
\hline \multicolumn{6}{|l|}{ cSCC } \\
\hline Locally advanced/metastatic cSCC & Cemiplimab-RWLC & PD-1 targeted antibody & 108 & 2018-09-28 & BLA761097 \\
\hline Recurrent/metastatic cSCC & Pembrolizumab & PD-1 targeted antibody & 105 & $2020-06-24$ & BLA125514 \\
\hline \multicolumn{6}{|l|}{$\mathrm{MCC}$} \\
\hline Metastatic MCC & Avelumab & $\begin{array}{l}\text { PD-L targeted anti- } \\
\text { body }\end{array}$ & 88 & $2017-03-23$ & BLA761049 \\
\hline Locally advanced/metastatic MCC & Pembrolizumab & PD-1 targeted antibody & 50 & 2018-12-19 & BLA125514 \\
\hline \multicolumn{6}{|l|}{ Tissue agnostic } \\
\hline $\begin{array}{l}\text { Unresectable/metastatic solid tumors-MSI high or } \\
\text { MMR-deficient }\end{array}$ & Pembrolizumab & PD-1 targeted antibody & 149 & $2017-05-23$ & BLA125514 \\
\hline Solid tumors-TMB High & Pembrolizumab & PD-1 targeted antibody & 102 & $2020-06-16$ & BLA125514 \\
\hline
\end{tabular}

found as a likely predictor of progression on immunotherapy and risk-benefit assessment is warranted for considerations of potential organ transplant loss (Table 2, See Potential Contraindications to Immunotherapy) [11].

Although they do not carry a labeled indication for NMSC, other ICIs may have efficacy and clinical benefit in patients with advanced CSCC as described in limited case series and case reports. Nivolumab, a monoclonal anti-PD-1 agent, was assessed in seven patients where a progressionfree survival (PFS) of 6-19.5 months was observed. A PR was noted in 5 patients treated with nivolumab and a CR was noted in a patient with poorly differentiated advanced CSCC treated with nivolumab and cetuximab [5, 14, 15]. Furthermore, several active clinical trials are assessing the response of nivolumab in the treatment of locally advanced and metastatic CSCC (Table 2, NCT04204837, NCT03834233)). A few case reports suggest activity in CSCC treated with ipilimumab, the first-in-class CTLA-4 inhibitor, approved by the FDA for metastatic melanoma in 2011. One patient with metastatic CSCC refractory to chemotherapy experienced a CR after 4 cycles of ipilimumab [16].

\section{Merkel Cell Carcinoma}

$\mathrm{MCC}$ is an aggressive neuroendocrine skin cancer, representing less than $1 \%$ of NMSCs. MCC is associated with ultraviolet radiation exposure, advanced age, immunosuppression, and the Merkel cell polyomavirus (MCPyV). Before the era of immunotherapy, advanced MCC was treated with chemotherapy. While the majority of patients show initial response to chemotherapy, the efficacy is short-lived (approximately 3 months), and there is no evidence of overall survival benefit [17]. Consequently, immunotherapy has emerged as the standard of care in first-line systemic therapy for advanced MCC.

Avelumab, an anti-PD-L1 agent, received accelerated FDA approval for the treatment of metastatic MCC in March of 2017. Part A of the JAVELIN (NCT02155647) Merkel 200 trial, an open-label, single-arm trial of patients with chemotherapy-refractory metastatic MCC (Table 1) reported an ORR of 33\% (23.3-43.8\%) with $11.4 \%$ of the subjects achieving a CR https://www.accessdata.fda.gov/ scripts/cder/daf/index.cfm?event=overview.process $\&$ ApplNo=761049, [18]. Grade 3-4 AEs were reported in $10.1 \%$ [19]. Interim analysis of part B of the JAVELIN Merkel 200 trial reported an ORR of $62.1 \%(95 \%$ CI, 42.3-79.3\%) in metastatic MCC patients treated with avelumab as first-line treatment [20]. Pembrolizumab also received an accelerated approval by the FDA in December of 2018 for the first-line treatment of recurrent locally advanced or metastatic MCC on the basis of KEYNOTE-017 (NCT02267603), a non-randomized, multicenter, open-label pivotal trial (Table 1) https://www.acces sdata.fda.gov/scripts/cder/daf/index.cfm?event=overview. process \&ApplNo $=125514$. The ORR was $56 \%(95 \%$ CI 41-70) and 24\% (95\% CI 13-38) of patients experienced a CR [21]. Furthermore, long-term observation studies of the expanded KEYNOTE-017 trial report continued durable disease control, a favorable OS and a manageable safety profile with pembrolizumab [22]. While currently 
not regulatory approved, the efficacy of nivolumab in advanced MCC has been assessed in the phase I/II CheckMate 358 (NCT02488759) trial and an ORR of 68\% was reported (Table 2) [23].

\section{Basal Cell Carcinoma}

$\mathrm{BCC}$ is the most common cancer and has an increasing incidence rate. Currently, two therapies targeting the hedgehog pathway are FDA-approved for the upfront treatment of recurrent, metastatic, or locally advanced BCC not amenable to surgery or radiation. The hedgehog signaling pathway is often dysregulated in BCCs through mutations in either PTCH1 or SMO genes. Vismodegib was the first hedgehog inhibitor (HHI) approved by the FDA in 2012 based on the phase II ERIVANCE (NCT00833417) trial (Table 1). An ORR of $47.6 \%$ for locally advanced BCC and $30 \%$ for metastatic BCC was observed at 12 months [24, 25]. At 39 months of follow-up, updated trial results reported an ORR of $60.3 \%$ and $48.5 \%$ for locally advanced and metastatic BCC, respectively [24].

Sonidegib is the second oral FDA-approved HHI for the upfront treatment of BCC (Table 1). Approved in 2015, sonidegib is indicated for the treatment of locally advanced BCC that has recurred following surgery or radiation therapy, or in candidates deemed ineligible for surgery or radiation. The phase II BOLT (NCT01327053) pivotal trial revealed an ORR of $56.1 \%$ with a median duration of response of 26.1 months and a $93.2 \%$ 2-year survival rate for locally advanced BCC. An ORR of $7.7 \%$ was reported for metastatic BCC (Table 1) [26].

There are currently no FDA approvals for first-line or upfront immunotherapy for BCCs that are locally advanced or metastatic. However, since BCC bears one of the highest TMBs, they are likely good candidates for treatment with ICI. Currently, pivotal trial data in the first-line setting are lacking, but several case reports with anti-PD-1 agents [28-30] and anti-CTLA-4 therapy [31] report activity and responses in advanced disease. A recent phase Ib study showed antitumor activity against advanced BCC with pembrolizumab. Seven patients received pembrolizumab plus vismodegib and nine patients received pembrolizumab alone [32]. The ORRs were $44 \%$ and $29 \%$ at 18 weeks and the PFS at 1 year were $62 \%$ and $83 \%$ for the monotherapy versus combination therapy cohorts, respectively (Table 2). Although not directly compared due to the non-randomized design of the study, the authors concluded combination therapy was not superior to monotherapy. The use of pembrolizumab in BCC has also been noted in 5 case reports with complete [30, 33] and partial [29, 34, 35] responses achieved, as well as a report of progressive disease of metastatic BCC bony lesions [30] on therapy. Nivolumab [28, 36] and cemiplimab [27] have also shown efficacy against advanced BCC. A patient with HHI-refractory recurrent metastatic BCC achieved a PR with cemiplimab [27]. Two patients with metastatic BCC were treated with nivolumab with one achieving a PR and PFS of 116 weeks [28] and the other patient achieved SD and PFS of 22 weeks [4].

Clinical trials investigating upfront ICIs in locally advanced, unresectable, or metastatic BCC are ongoing. This includes a non-randomized open-label Phase II trial (NCT03521830) where patients receive nivolumab alone or nivolumab in combination with ipilimumab. Additionally, there is a phase I/II trial (NCT02690948) investigating pembrolizumab monotherapy versus pembrolizumab plus vismodegib (Table 2).

In February of 2021, cemiplimab received an accelerated FDA approval for the treatment of patients with locally advanced or metastatic BCC who experienced progression of disease on HHI or for HHI-therapy intolerant patients. Approval was based on the results of a phase II (NCT03132636) open-label, multicenter, non-randomized trial (Table 1). An ORR of $21 \%$ and $29 \%$ were reported for patients with metastatic and locally advanced BCC, respectively https://www.accessdata.fda.gov/scripts/cder/daf/ index.cfm? event $=$ overview. process \& $\mathrm{App} 1 \mathrm{No}=761097$. With a recent FDA-approval for immunotherapy in the HHIrefractory setting, continued studies and trials (Table 2) are likely to lead to an expansion in FDA-approved indications in advanced BCC.

\section{Rare Cutaneous Adnexal Carcinomas}

Cutaneous adnexal carcinomas (CACs) represent a heterogenous group of rare skin cancers including porocarcinoma and sebaceous carcinoma with limited effective systemic therapy options for advanced disease [37]. These malignancies display differentiation toward skin-primary adnexal structures such as eccrine or apocrine glands. Limited case reports in the literature have illustrated tumor response to ICIs in various CACs. For example, a patient with metastatic porocarcinoma achieved a clinical and radiological CR when treated with pembrolizumab [38] and a patient with widely metastatic sebaceous carcinoma experienced a near CR with pembrolizumab [39] CACs [40] and sebaceous carcinomas with high PD-L1 expression levels have been previously reported [41].

There are currently no FDA-approved immunotherapeutics for CACs specifically. However, the regulatory approval of tumor tissue-agnostic indications for pembrolizumab may play a role in the management of advanced CACs (Table 1). In May of 2017, the FDA approved pembrolizumab for the treatment of unresectable or metastatic, microsatellite instability-high (MSI-H) or mismatch repair (MMR) deficient tumors that have progressed following prior treatment. The accelerated approval was based on 
the trial results of patients with MSI-H or MMR-deficient cancers treated with pembrolizumab identified across five clinical trials (NCT01876511, NCT02460198, NCT01848834, NCT02054806, NCT02628067; Table 1). An ORR of $39.6 \%$ with $7.4 \%$ achieving a CR was reported https://www.accessdata.fda.gov/scripts/cder/daf/index. $\mathrm{cfm}$ ? event $=$ overview.process $\& \mathrm{ApplNo}=125514$. The rationale for assessing MSI status in sebaceous carcinoma is supported by data reporting germline variants in the MMR genes MSH2, MSH6, and MLH1 in $8-29 \%$ of patients [43-45]. Individuals with sebaceous carcinomas, non-polyposis colorectal cancer, and germline loss of MMRs are characterized as having Muir-Torre syndrome (OMIM 158,320) and their tumors demonstrate microsatellite instability [46]. Furthermore, in June of 2020, the FDA approved a second tumor tissue-agnostic indication for pembrolizumab for unresectable or metastatic tumor mutational burden-high (TMB-H) $[\geq 10$ mutations/ megabase] solid tumors that have progressed following prior treatment (Table 1) https://www.accessdata.fda. gov/scripts/cder/daf/index.cfm?event=overview.proce ss\&ApplNo $=125514$. The efficacy was investigated in the open-label, multicenter, non-randomized KEYNOTE-158 (NCT02628067) trial where an ORR of $29 \%$ and $37 \%$ was reported for the TMB $\geq 10$ mutations/megabase cohort and $\mathrm{TMB} \geq 13$ mutations/megabase cohort, respectively. For advanced and metastatic CACs, tumors may be assessed for MSI, MMR status, and TMB levels for potential treatment with pembrolizumab since no satisfactory treatment options are available. Future studies are required to ascertain clinical benefit and response to pembrolizumab and other ICIs for the treatment of advanced CACs, especially since no CACs or NMSCs were included in the tissueagnostic trials.

\section{Immunotherapy Rechallenge}

Despite the success of upfront ICI in advanced NMSCs, many patients develop resistance to immunotherapy after an initial response or do not have tumor response at all. The use of sequential ICIs or ICIs in combinatorial regimens represent a potentially promising approach for patients that do not have durable benefit from ICI. For example, a multi-institutional retrospective case series $(N=13)$ assessed rescue therapy for ICI-refractory MCC (Table 2) [47]. Second-line ICI treatment in MCC patients that progressed on earlier ICI treatment revealed an ORR of $31 \%$. In addition, a case report details a patient with metastatic MCC who progressed on pembrolizumab monotherapy 10 months after initiating ICI [48]. However, a durable response and CR was achieved when treated with concurrent radiation with pembrolizumab. Further investigation is required to determine if second-line ICIs or combination radiation therapy might improve systemic responses to NMSC tumors initially refractory to ICI.

\section{Adjuvant and Neoadjuvant Immunotherapy}

Although ICI treatment strategies for metastatic disease have been successful, therapeutic approaches to preclude the development of advanced disease in high-risk NMSC remain an unmet need. Improved recurrence-free survival (RFS) has been shown with ICI use in resected melanoma [49-51]. and similar strategies are being investigated in NMSC (Table 2). A phase III (NCT03969004) trial is randomizing patients with high-risk CSCC after surgery and radiation therapy to either treatment with adjuvant cemiplimab or placebo. A phase II trial (NCT03057613) is assessing patients with resected CSCC of the head and neck treated with radiation therapy in combination with adjuvant pembrolizumab. A phase III double-blinded placebo-controlled study (NCT03833167) is assessing adjuvant pembrolizumab in patients with resected high-risk CSCC (Table 2).

There are also randomized trials examining anti-PD-1 or anti-PD-L1 inhibitors in the adjuvant setting for MCC. The phase III STAMP trial (NCT03712605) is randomizing patients with stage I-IIIB MCC. MCC patients with stage I disease and negative sentinel lymph nodes are excluded from enrollment. Following resection, patients are treated with either pembrolizumab or standard-of-care observation. Avelumab is also being investigated in the adjuvant setting. The phase III ADAM trial (NCT03271372) is an ongoing, multicenter, randomized, double-blind study comparing adjuvant avelumab versus placebo in patients with MCC that has metastasized to the lymph nodes and who have undergone surgery and/or radiation. The phase II I-MAT trial (NCT04291885) is a prospective, placebo-controlled study for patients with stage I-III MCC also aiming to explore the role of avelumab in the adjuvant setting. Nivolumab is being investigated in the adjuvant setting in the phase II ADMEC-O trial (NCT02196961). Enrolled patients receive nivolumab monotherapy in completely resected MCC versus standard-of-care observation. Adjuvant ipilimumab monotherapy failed to demonstrate prevention of disease progression in the adjuvant setting for resected MCC and resulted in pronounced AEs [52]. A phase I trial (NCT03798639) is randomizing patients with stage IIIA/B MCC to receive either nivolumab and radiation therapy or nivolumab in combination with ipilimumab to assess these two different immunotherapy regimens in the adjuvant setting (Table 2).

While the ability to offer patients many clinical trial options for adjuvant ICIs is important, it poses challenges as well. Due to experiencing relatively slow accrual, the 
Table 2 Real-world evidence and active clinical trials using ICI in NMSCs

\begin{tabular}{|c|c|c|c|}
\hline Upfront setting & Study design & Agent(s) & Published findings \\
\hline \multicolumn{4}{|l|}{ CSCC } \\
\hline Unresectable & $\begin{array}{l}\text { Phase II [CARSKIN] } \\
\text { (NCT02883556) }\end{array}$ & Pembrolizumab & $41 \%$ ORR [10] \\
\hline Unresectable/metastatic & Phase II (NCT02721732) & Pembrolizumab & - \\
\hline Locally adv/recurrent & Phase II (NCT02964559) & Pembrolizumab & - \\
\hline Locally adv/metastatic & Phase II (NCT03284424) & Pembrolizumab & - \\
\hline Locally adv/metastatic & $\begin{array}{l}\text { Retrospective, single institution } \\
(N=76)\end{array}$ & $\begin{array}{l}\text { Pembrolizumab, cemiplimab, } \\
\text { nivolumab }\end{array}$ & $34 \%$ ORR [11] \\
\hline Locally adv/metastatic & $\begin{array}{l}\text { Retrospective, single institution } \\
(N=61)\end{array}$ & $\begin{array}{l}\text { Pembrolizumab, cemiplimab, } \\
\text { nivolumab }\end{array}$ & $31.5 \%$ ORR [12] \\
\hline Locally adv/metastatic & $\begin{array}{l}\text { Retrospective, multi-institution } \\
(N=46)\end{array}$ & $\begin{array}{l}\text { Pembrolizumab, cemiplimab, } \\
\text { nivolumab }\end{array}$ & $58.7 \%$ ORR [13] \\
\hline Locally adv/metastatic & $\begin{array}{l}\text { Interventional clinical trial } \\
\text { (NCT03834233) }\end{array}$ & Nivolumab & - \\
\hline Locally adv/metastatic & $\begin{array}{l}\text { Interventional clinical trial } \\
\text { (NCT04204837) }\end{array}$ & Nivolumab & - \\
\hline \multicolumn{4}{|l|}{ MCC } \\
\hline Virus-associated diseases (MCC) & $\begin{array}{l}\text { Phase I/II [CheckMate 358] } \\
\text { (NCT02488759) }\end{array}$ & Nivolumab & $68 \%$ ORR [23] \\
\hline $\begin{array}{l}\text { Advanced, refractory to initial } \\
\text { ICI therapy }\end{array}$ & $\begin{array}{l}\text { Retrospective, multi-institution } \\
(N=13)\end{array}$ & $\begin{array}{l}\text { Pembrolizumab, avelumab, } \\
\text { nivolumab }\end{array}$ & $31 \%$ ORR [47] \\
\hline \multicolumn{4}{|l|}{$\mathrm{BCC}$} \\
\hline Advanced BCC & Phase $\mathrm{Ib}(N=16)(\mathrm{NCT} 02690948)$ & $\begin{array}{l}\text { Pembrolizumab + vismodegib or } \\
\text { pembrolizumab alone }\end{array}$ & $\begin{array}{l}\text { 29\% ORR combination [32]; } \\
\text { 44\% ORR pembrolizumab } \\
\text { [32] }\end{array}$ \\
\hline Locally adv/metastatic & Phase II(NCT03521830) & $\begin{array}{l}\text { Nivolumab + ipilimumab or } \\
\text { nivolumab alone }\end{array}$ & - \\
\hline Adjuvant setting & Study design & Agent(s) & Published findings \\
\hline \multicolumn{4}{|l|}{$\mathrm{CSCC}$} \\
\hline Resected H\&N CSCC & Phase II(NCT03057613) & $\begin{array}{l}\text { Adjuvant pembrolizumab with } \\
\text { postoperative XRT }\end{array}$ & - \\
\hline Resected high-risk CSCC & Phase III(NCT03833167) & Adjuvant pembrolizumab & - \\
\hline Resected high-risk CSCC & Phase III(NCT03969004) & Adjuvant cemiplimab or placebo & - \\
\hline \multicolumn{4}{|l|}{ MCC } \\
\hline Resected stage I-IIIB MCC & Phase III [STAMP](NCT03712605) & Pembrolizumab or observation & - \\
\hline $\begin{array}{l}\text { Resected MCC with nodal } \\
\text { metastasis }\end{array}$ & Phase III [ADAM](NCT03271372) & Avelumab or placebo & - \\
\hline Resected stage I-III MCC & Phase II [I-MAT](NCT04291885) & Avelumab or placebo & - \\
\hline Resected MCC & $\begin{array}{l}\text { Phase II [ADEMC-O] } \\
\text { (NCT02196961) }\end{array}$ & Nivolumab or observation & - \\
\hline Resected stage IIIA-IIIB MCC & Phase I(NCT03798639) & $\begin{array}{l}\text { Nivolumab + radiation or } \\
\text { nivolumab + ipilimumab }\end{array}$ & - \\
\hline \multicolumn{4}{|l|}{$\mathrm{BCC}$} \\
\hline Advanced BCC of H\&N & Phase Ib(NCT04323202) & $\begin{array}{l}\text { Neoadjuvant-adjuvant pembroli- } \\
\text { zumab }\end{array}$ & - \\
\hline Neoadjuvant setting & Study design & Agent(s) & Published findings \\
\hline \multicolumn{4}{|l|}{$\mathrm{CSCC}$} \\
\hline Stage III-IV H\&N CSCC & Phase II(NCT03565783) & Neoadjuvant cemiplimab & $30 \%$ ORR $[57 \bullet]$ \\
\hline \multicolumn{4}{|l|}{$\mathrm{MCC}$} \\
\hline Stage IIA-IV MCC & $\begin{array}{l}\text { Phase I/II [CheckMate 358] } \\
\text { (NCT02488759) }\end{array}$ & Neoadjuvant nivolumab & $47.2 \%$ pCR $[58 \bullet]$ \\
\hline
\end{tabular}


Table 2 (continued)

BCC

feasibility of trials in this setting are being re-examined due to the competing trial aspect. The challenge to recruit to adjuvant trials is further compounded by the growth of neoadjuvant strategies. Administration of ICI prior to resection of high-risk lesions has the potential to be advantageous for several reasons, though these studies often have significant overlap in the patient population targeted by adjuvant trials. Nevertheless, despite these logistical complexities, neoadjuvant treatment is emerging as a very active area of investigation as upfront ICI may not only reduce tumor burden and facilitate resection, but possibly also enhance tumor-specific immune responses [53]. Furthermore, pathological examination of neoadjuvantly treated tumors at the time of resection offers the potential to identify biomarkers of response and survival. Although not without their challenges, results from early neoadjuvant ICI strategies in melanoma have produced encouraging results [54-56]. In NMSC, the phase II open-label (NCT03565783) trial assessed the role of cemiplimab in the neoadjuvant setting for stage III-IV CSCC of the head and neck (Table 2). Early reports reveal an ORR of $30 \%$ with a pathological complete response (pCR) achieved in 55\% of patients.[57•]

Nivolumab was assessed in the neoadjuvant setting for patients with resectable stage IIA-IV MCC in the CHECKMATE 358 Trial (NCT02488759).[58•] A pCR was achieved in $47.2 \%$ of the cohort and responses were independent of TMB, MCPyV, or PD-L1 status. During the observation study period, no patient with a pCR had tumor relapse. A pathological complete response to neoadjuvant avelumab was also reported in a patient with MCC [59]. In BCC, a single-arm phase IB (NCT04323202) neoadjuvant-adjuvant study will investigate pembrolizumab therapy administered prior to and following resection in advanced disease of the head and neck.

\section{Potential Contraindications to Immunotherapy}

Additional safety profile considerations of ICIs are warranted since patients with advanced NMSCs are often immunosuppressed and/or of advanced age. Patients with a long-standing history of immunosuppressive medications, hematological malignancy, or HIV require consideration for ICI since these cohorts are often excluded from trials. For example, the use of ICIs in transplant recipients may be challenging due to enhanced T-cell activation potentially leading to allograft rejection [60]. Current trial data is limited and data from case series and reports reveal significant risk of allograft rejection but consistent durable disease control [61-63]. Further studies are needed to determine the safety profile of ICIs in immunosuppressed populations with advanced NMSCs. A specific emphasis on research efforts aimed at determining suitable therapy regimens optimized for graft preservation without reducing ICI antitumor activity would be beneficial to this group. Data from case reports and case series reveal responses to ICI in immunosuppressed NMSC patients may be comparable to immunocompetent patients [11, 12]. Notably, a recent case series reported a PR of advanced CSCC in an HIV patient [4].

\section{Challenges and Future Directions}

Despite exceptional responses to ICIs in NMSCs, the associated immune-related AEs (irAE) require careful monitoring. Thus, clinical research efforts should aim to identify new ICI regimens that enhance tumor response while reducing toxicity and irAE severity. In addition, continued efforts to identify predictive and prognostic biomarkers of response and resistance to anti-CTLA-4 and anti-PD-L1/PD- 1 therapies may help identify patients with NMSC that are likely to benefit from therapy. A possible cause of failure to respond to ICIs is the lack of costimulatory signals in the tumor microenvironment. Current active research is focused on promoting response by utilizing costimulatory checkpoint agonists and innate immune targets such as OX40 agonists, oncolytic viruses, and STING/Toll-like receptor (TLR) agonists currently under investigation in patients with multiple solid tumors, including advanced NMSC. Furthermore, specific assessment of the role of ICIs in advanced CACs are required.

Lastly, we must consider barriers to enrollment to ongoing clinical studies - such as trial rollout and study designto maintain continued progress. For example, launching synchronous studies in the neoadjuvant and adjuvant settings simultaneously can impair enrollment to both, as there is often significant overlap in these patient populations. Furthermore, we have found that certain elements of trial design have had substantial effects on recruitment. For example, enrolling patients on adjuvant ICI trials following adjuvant radiotherapy has proved challenging. Patients have expressed a preference to undergo only one therapy in the adjuvant setting and "reserve" immunotherapy for the relapsed setting. Similarly, we have found that the decision to incorporate a placebo as compared to a "standard-of-care" comparator arm has greatly affected trial participation, especially in the 
time of the COVID-19 pandemic. While placebo arms play an important role in decreasing bias and confounders, they can negatively affect recruitment. Patients have been less enthusiastic about returning to clinic regularly for prolonged periods of time (e.g., every 2-6 weeks for an entire year) if there is a significant chance they are not going to receive the investigational agent. This is of particular concern in the NMSC population, which on average, is typically older with numerous comorbidities and increasingly reliant on others for transportation to clinic.

Of course, these challenges are not unique to the NMSC patient population. However, in the absence of a high-level assessment of the trial landscape, the NMSC field may turn one of its greatest assets into its biggest liability. The robust clinical activity of immunotherapy in NMSC has attracted attention by a great number of pharmaceutical companies. Patients with NMSC can provide a relatively straight-forward path to a new Biological License Application (BLA) or a supplemental efficacy indication. Consequently, the number of trials in the NMSC space has increased significantly over the last few years and that growth is likely to continue in the years to come. And while a multitude of therapeutic trial options has a theoretical advantage, a bigger concern is competition of these trials for a relatively stable patient population. Thus, without improved communication between sponsors, academicians and patient advocacy groups, we run the real risk of opening trials that fail to meet their enrollment goals and primary endpoints.

\section{Conclusions}

Anti-PD-1/PD-L1 and CTLA-4 inhibitors have improved survival for many advanced NMSC patients. Immunotherapy is playing an increasingly critical role in the management of advanced disease and is considered standard of care for upfront systemic therapy in locally advanced and unresectable MCC and CSCC, and more recently in HHI-refractory BCC. Potentially, ICIs and other forms of immunotherapy may also play an important role in the neoadjuvant and adjuvant settings as well, with several trials currently underway. Continued evaluation of the clinical trial landscape is needed to optimize enrollment and ensure perpetual innovation.

\section{Declarations}

Conflict of Interest Sophia Z. Shalhout declares that she has no conflict of interest. Kevin S. Emerick has received honoraria for participating on advisory boards for Regeneron, Sanofi Genzyme, and Jounce Therapeutics. Howard L. Kaufman is an employee of Immuneering Corporation. David M. Miller honoraria for participating on advisory boards for Checkpoint Therapeutics, EMD Serono, Pfizer, Merck, Regeneron, and Sanofi Genzyme.

\section{References}

Papers of particular interest, published recently, have been highlighted as:

- Of importance

$\bullet$ Of major importance

1. Global Burden of Disease Cancer C, Fitzmaurice C, Abate D, et al. Global, regional, and national cancer incidence, mortality, years of life lost, years lived with disability, and disabilityadjusted life-years for 29 cancer groups, 1990 to 2017: a systematic analysis for the global burden of disease study. JAMA Oncol. 2019;5(12):1749-68.

2. Harms PW. Update on Merkel cell carcinoma. Clin Lab Med. 2017;37(3):485-501.

3. Karia PS, Han J, Schmults CD. Cutaneous squamous cell carcinoma: estimated incidence of disease, nodal metastasis, and deaths from disease in the United States, 2012. J Am Acad Dermatol. 2013;68(6):957-66.

4. Borradori L, Sutton B, Shayesteh P, Daniels GA. Rescue therapy with anti-programmed cell death protein 1 inhibitors of advanced cutaneous squamous cell carcinoma and basosquamous carcinoma: preliminary experience in five cases. Br J Dermatol. 2016;175(6):1382-6.

5. Tran DC, Colevas AD, Chang AL. Follow-up on programmed cell death 1 inhibitor for cutaneous squamous cell carcinoma. JAMA Dermatol. 2017;153(1):92-4.

6.•• Migden MR, Rischin D, Schmults CD, et al. PD-1 blockade with cemiplimab in advanced cutaneous squamous-cell carcinoma. $\mathrm{N}$ Engl J Med. 2018;379(4):341-51.. (The seminal results of the expansion cohort of the phase I and phase II pivotal trials assesing cemiplimab for advanced and metastatic cutaneous squamous cell carcinoma lead to the first FDA-approved anti-PD-1 agent for upfront therapy in this disease.)

7. Guminski AD, Lim AML, Khushalani NI, et al. Phase 2 study of cemiplimab, a human monoclonal anti-PD-1, in patients with metastatic cutaneous squamous cell carcinoma (mCSCC; Group 1): 12-month follow-up [abstract]. J Clin Oncol. 2019;37(15 suppl):9526.

8. Owonikoko TKPK, Johnson ML, et al. Phase 1 study of cemiplimab, a human monoclonal anti-PD-1, in patients with unresectable locally advanced or metastatic cutaneous squamous cell carcinoma (CSCC): final efficacy and safety data [abstract]. J Clin Oncol. 2018;36(15 suppl):9557.

9. Grob JJ, Gonzalez R, Basset-Seguin N, et al. Pembrolizumab monotherapy for recurrent or metastatic cutaneous squamous cell carcinoma: a single-arm phase II trial (KEYNOTE-629). J Clin Oncol. 2020;38(25):2916-25.. (The results from the phase II pivotal trial assesing pembrolizumab for the treatment of cutaneous squamous cell carcinoma lead to the second FDA approval of an anti-PD-1 agent for first-line therapy in advanced CSCC not amenable to curative surgery or radiation.)

10. Maubec E, Boubaya M, Petrow P, et al. Phase II study of pembrolizumab as first-line, single-drug therapy for patients with unresectable cutaneous squamous cell carcinomas. J Clin Oncol. 2020;38(26):3051-61.

11. Shalhout SZ, Park JC, Emerick KS, Sullivan RJ, Kaufman HL, Miller DM. Real-world assessment of response to antiprogrammed cell death 1 therapy in advanced cutaneous squamous cell carcinoma. J Am Acad Dermatol. 2021;19:S01909622(21)00197-3. https://doi.org/10.1016/j.jaad.2021.01.048.

12. Hanna GJ, Ruiz ES, LeBoeuf NR, et al. Real-world outcomes treating patients with advanced cutaneous squamous cell 
carcinoma with immune checkpoint inhibitors (CPI). Br J Cancer. 2020;123(10):1535-42.

13. Salzmann M, Leiter U, Loquai C, et al. Programmed cell death protein 1 inhibitors in advanced cutaneous squamous cell carcinoma: real-world data of a retrospective, multicenter study. Eur J Cancer. 2020;138:125-32.

14. Chen A, Ali N, Boasberg P, Ho AS. Clinical Remission of Cutaneous Squamous Cell Carcinoma of the Auricle with Cetuximab and Nivolumab. J Clin Med. 2018;7(1):10. https://doi.org/10. 3390/jcm7010010.

15. Blum V, Muller B, Hofer S, et al. Nivolumab for recurrent cutaneous squamous cell carcinoma: three cases. Eur J Dermatol. 2018;28(1):78-81.

16. Day F, Kumar M, Fenton L, Gedye C. Durable response of metastatic squamous cell carcinoma of the skin to ipilimumab immunotherapy. J Immunother. 2017;40(1):36-8.

17. Bichakjian CK, Olencki T, Aasi SZ, et al. Merkel cell carcinoma, Version 1.2018, NCCN Clinical Practice Guidelines in Oncology. J Natl Compr Canc Netw. 2018;16(6):742-74.

18. Kaufman HL, Russell J, Hamid O, et al. Avelumab in patients with chemotherapy-refractory metastatic Merkel cell carcinoma: a multicentre, single-group, open-label, phase 2 trial. Lancet Oncol. 2016;17(10):1374-85.

19. Kaufman HL, Russell JS, Hamid O, et al. Updated efficacy of avelumab in patients with previously treated metastatic Merkel cell carcinoma after $>/=1$ year of follow-up: JAVELIN Merkel 200, a phase 2 clinical trial. J Immunother Cancer. 2018;6(1):7.

20. D'Angelo SP, Russell J, Lebbe C, et al. Efficacy and safety of first-line avelumab treatment in patients with stage IV metastatic Merkel cell carcinoma: a preplanned interim analysis of a clinical trial. JAMA Oncol. 2018;4(9):180077.

21. Nghiem PT, Bhatia S, Lipson EJ, et al. PD-1 blockade with pembrolizumab in advanced Merkel-cell carcinoma. N Engl J Med. 2016;374(26):2542-52.

22. Nghiem P, Bhatia S, Lipson EJ, et al. Durable tumor regression and overall survival in patients with advanced Merkel cell carcinoma receiving pembrolizumab as first-line Therapy. J Clin Oncol. 2019;37(9):693-702.

23. Topalian SLBS, Hollebecque A, et al. Non-comparative, openlabel, multiple cohort, phase $1 / 2$ study to evaluate nivolumab (NIVO) in patients with virus-associated tumors (CheckMate 358): efficacy and safety in Merkel cell carcinoma (MCC) [abstract]. Cancer Res. 2017;77(13 suppl):CT074.

24. Sekulic A, Migden MR, Basset-Seguin N, et al. Long-term safety and efficacy of vismodegib in patients with advanced basal cell carcinoma: final update of the pivotal ERIVANCE BCC study. BMC Cancer. 2017;17(1):332.

25. Sekulic A, Migden MR, Lewis K, et al. Pivotal ERIVANCE basal cell carcinoma (BCC) study: 12-month update of efficacy and safety of vismodegib in advanced BCC. J Am Acad Dermatol. 2015;72(6):1021-1026 e1028.

26. Lear JT, Migden MR, Lewis KD, et al. Long-term efficacy and safety of sonidegib in patients with locally advanced and metastatic basal cell carcinoma: 30-month analysis of the randomized phase 2 BOLT study. J Eur Acad Dermatol Venereol. 2018;32(3):372-81.

27. Falchook GS, Leidner R, Stankevich E, et al. Responses of metastatic basal cell and cutaneous squamous cell carcinomas to anti-PD1 monoclonal antibody REGN2810. J Immunother Cancer. 2016;4:70.

28. Ikeda S, Goodman AM, Cohen PR, Jensen TJ, Ellison CK, Frampton G, Miller V, Patel SP, Kurzrock R. Metastatic basal cell carcinoma with amplification of PD-L1: exceptional response to anti-PD1 therapy. NPJ Genom Med. 2016;1:16037. https://doi.org/10.1038/npjgenmed.2016.37.
29. Lipson EJ, Lilo MT, Ogurtsova A, et al. Basal cell carcinoma: PD-L1/PD-1 checkpoint expression and tumor regression after PD-1 blockade. J Immunother Cancer. 2017;5:23.

30. Cannon JGD, Russell JS, Kim J, Chang ALS. A case of metastatic basal cell carcinoma treated with continuous PD-1 inhibitor exposure even after subsequent initiation of radiotherapy and surgery. JAAD Case Rep. 2018;4(3):248-50.

31. Mohan SV, Kuo KY, Chang AL. Incidental regression of an advanced basal cell carcinoma after ipilimumab exposure for metastatic melanoma. JAAD Case Rep. 2016;2(1):13-5.

32. Chang ALS, Tran DC, Cannon JGD, et al. Pembrolizumab for advanced basal cell carcinoma: an investigator-initiated, proofof-concept study. J Am Acad Dermatol. 2019;80(2):564-6.

33. Moreira A, Kirchberger MC, Toussaint F, Erdmann M, Schuler G, Heinzerling L. Effective anti-programmed death-1 therapy in a SUFU-mutated patient with Gorlin-Goltz syndrome. Br J Dermatol. 2018;179(3):747-9.

34. Winkler JK, Schneiderbauer R, Bender C, et al. Anti-programmed cell death-1 therapy in nonmelanoma skin cancer. Br J Dermatol. 2017;176(2):498-502.

35. Fischer S, Hasan Ali O, Jochum W, Kluckert T, Flatz L, Siano M. Anti-PD-1 therapy leads to near-complete remission in a patient with metastatic basal cell carcinoma. Oncol Res Treat. 2018;41(6):391-4.

36. Cohen PR, Kato S, Goodman AM, Ikeda S, Kurzrock R. Appearance of New Cutaneous Superficial Basal Cell Carcinomas during Successful Nivolumab Treatment of Refractory Metastatic Disease: Implications for Immunotherapy in Early Versus Late Disease. Int J Mol Sci. 2017;18(8):1663. https:// doi.org/10.3390/ijms18081663.

37. Martinez SR, Barr KL, Canter RJ. Rare tumors through the looking glass: an examination of malignant cutaneous adnexal tumors. Arch Dermatol. 2011;147(9):1058-62.

38. Lee KA, Cioni M, Robson A, Bataille V. Metastatic porocarcinoma achieving complete radiological and clinical response with pembrolizumab. BMJ Case Rep. 2019;12(9):e228917. https://doi.org/10.1136/bcr-2018-228917.

39. Domingo-Musibay E, Murugan P, Giubellino A, et al. Near complete response to pembrolizumab in microsatellite-stable metastatic sebaceous carcinoma. J Immunother Cancer. 2018;6(1):58.

40. Duverger L, Osio A, Cribier B, et al. Heterogeneity of PD-L1 expression and CD8 tumor-infiltrating lymphocytes among subtypes of cutaneous adnexal carcinomas. Cancer Immunol Immunother. 2019;68(6):951-60.

41. Kandl TJ, Sagiv O, Curry JL, et al. High expression of PD-1 and PD-L1 in ocular adnexal sebaceous carcinoma. Oncoimmunology. 2018;7(9):e1475874.

42. Roberts ME, Riegert-Johnson DL, Thomas BC, et al. Screening for Muir-Torre syndrome using mismatch repair protein immunohistochemistry of sebaceous neoplasms. J Genet Couns. 2013;22(3):393-405.

43. Roberts ME, Riegert-Johnson DL, Thomas BC, et al. A clinical scoring system to identify patients with sebaceous neoplasms at risk for the Muir-Torre variant of Lynch syndrome. Genet Med. 2014;16(9):711-6.

44. Everett JN, Raymond VM, Dandapani M, et al. Screening for germline mismatch repair mutations following diagnosis of sebaceous neoplasm. JAMA Dermatol. 2014;150(12):1315-21.

45. Nguyen CV, Gaddis KJ, Stephens MR, Seykora JT, Chu EY. An intrapatient concordance study of mismatch repair protein immunohistochemical staining patterns in patients with MuirTorre syndrome. JAMA Dermatol. 2020;156(6):676-80.

46. Sargen MR, Starrett GJ, Engels EA, Cahoon EK, Tucker MA, Goldstein AM. Sebaceous Carcinoma epidemiology 
and genetics: emerging concepts and clinical implications for screening, prevention, and treatment. Clin Cancer Res. 2021;27(2):389-93.

47. LoPiccolo J, Schollenberger MD, Dakhil S, et al. Rescue therapy for patients with anti-PD-1-refractory Merkel cell carcinoma: a multicenter, retrospective case series. J Immunother Cancer. 2019;7(1):170.

48. Bloom BC, Augustyn A, Pezzi TA, et al. Rescue of immunotherapy-refractory metastatic merkel cell carcinoma with conventionally fractionated radiotherapy and concurrent pembrolizumab. Front Oncol. 2019;9:223.

49. Weber J, Mandala M, Del Vecchio M, et al. Adjuvant nivolumab versus ipilimumab in resected stage III or IV melanoma. N Engl J Med. 2017;377(19):1824-35.

50. Eggermont AM, Chiarion-Sileni V, Grob JJ, et al. Adjuvant ipilimumab versus placebo after complete resection of highrisk stage III melanoma (EORTC 18071): a randomised, doubleblind, phase 3 trial. Lancet Oncol. 2015;16(5):522-30.

51. Eggermont AMM, Blank CU, Mandala M, et al. Adjuvant pembrolizumab versus placebo in resected stage III melanoma. N Engl J Med. 2018;378(19):1789-801.

52. Becker JC, Hassel JC, Menzer C, Kähler KC, Eigentler TK, et al. DeCOG/ADO. J Clin Oncol. 2018;36:9527-9527.

53. Liu J, Blake SJ, Yong MC, et al. Improved efficacy of neoadjuvant compared to adjuvant immunotherapy to eradicate metastatic disease. Cancer Discov. 2016;6(12):1382-99.

54. Rozeman EA, Menzies AM, van Akkooi ACJ, et al. Identification of the optimal combination dosing schedule of neoadjuvant ipilimumab plus nivolumab in macroscopic stage III melanoma (OpACIN-neo): a multicentre, phase 2, randomised, controlled trial. Lancet Oncol. 2019;20(7):948-60.

55. Huang AC, Orlowski RJ, Xu X, et al. A single dose of neoadjuvant PD-1 blockade predicts clinical outcomes in resectable melanoma. Nat Med. 2019;25(3):454-61.

56. Amaria RN, Reddy SM, Tawbi HA, et al. Neoadjuvant immune checkpoint blockade in high-risk resectable melanoma. Nat Med. 2018;24(11):1649-54.

57. Gross N. Ferrarotto R, Nagarajan P, Bell D, El-Naggar A, Johnson JM, Yuan Y, Glisson B, Wong M, Rosenthal D, Esmaeli B, Migden MR, Wargo J, Weber RS, Myers J. LBA74 - Phase II study of neoadjuvant cemiplimab prior to surgery in patients with stage III/IV (M0) cutaneous squamous cell carcinoma of the head and neck (CSCC-HN). Ann Oncol. 2019;30:v910. https:// doi.org/10.1093/annonc/mdz394.071. This abstract reports the promising early results of the phase II trial assessing neoadjuvant cemiplimab in patients with stage III/IV CSCC. An ORR of $30 \%$ and a pathological complete response in $55 \%$ of patients was achieved.

58. Topalian SL, Bhatia S, Amin A, et al. Neoadjuvant Nivolumab for Patients With Resectable Merkel Cell Carcinoma in the CheckMate 358 Trial. J Clin Oncol. 2020;38(22):2476-87.. (The seminal CheckMate 358 trial assessed nivolumab in the neoadjuvant setting for patients with resectable Stage IIA-IV MCC and reported a pCR of $47.2 \%$ in the cohort, suggesting a valuable role for immunotherapy in the neoadjuvant setting for MCC.)

59. Abdallah N, Nagasaka M, Chowdhury T, Raval K, Hotaling J, Sukari A. Complete response with neoadjuvant avelumab in Merkel cell carcinoma - a case report. Oral Oncol. 2019;99:104350.

60. Chae YK, Galvez C, Anker JF, Iams WT, Bhave M. Cancer immunotherapy in a neglected population: the current use and future of T-cell-mediated checkpoint inhibitors in organ transplant patients. Cancer Treat Rev. 2018;63:116-21.

61. Cippa PE, Schiesser M, Ekberg H, et al. Risk stratification for rejection and infection after kidney transplantation. Clin J Am Soc Nephrol. 2015;10(12):2213-20.

62. Sadaat M, Jang S. Complete tumor response to pembrolizumab and allograft preservation in renal allograft recipient on immunosuppressive Therapy. J Oncol Pract. 2018;14(3):198-9.

63. Lipson EJ, Bagnasco SM, Moore J Jr, et al. Tumor regression and allograft rejection after administration of anti-PD-1. N Engl J Med. 2016;374(9):896-8.

Publisher's Note Springer Nature remains neutral with regard to jurisdictional claims in published maps and institutional affiliations. 